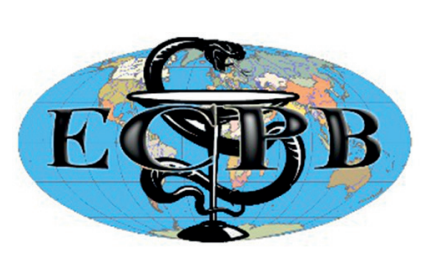

“ЕКСПЕРИМЕНТАЛЬНА ТА КЛІНІЧНА ФІЗІОЛОГІЯ І БІОХІМІЯ” "EXPERIMENTAL AND CLINICAL PHYSIOLOGY AND BIOCHEMISTRY" Науково-практичний журнал/Scientific-practical journal

ECPB 2019, 3(87): 12-22. https://doi.org/

\title{
Factor Analysis of the Information Field of the Neuroendocrine-Immune Complex and Metabolism in Female Rats
}

\author{
Y.V. ZAVIDNYUK ${ }^{1,2}$, O.I. MEL'NYK ${ }^{3}$, O.G. MYSAKOVETS' ${ }^{31}$
}

${ }^{1} I$.Y. Horbachevs'kyi National Medical University, Ternopil', Ukraine

zavidnyukyv@tdmu.edu.ua

${ }^{2}$ Ukrainian Scientific Research Institute of Medicine for Transport, Odesa, Ukraine ${ }^{3}$ Danylo Halyts'ky National Medical University, L'viv, Ukraine

\section{oksana@meduniv.lviv.ua}

Introduction. Despite considerable informativeness, factor analysis in biomedical research is still rarely used. Therefore, we set out to introduce our colleagues to the theoretical foundations of factor analysis and to demonstrate its application in our own material.

According to the theory of factor analysis [1], it is considered that the observed parameters (variables) are a linear combination of some latent (hypothetical, unobservable) factors. In other words, the factors are hypothetical, not directly measured, hidden variables, in terms of which the measured variables are described. Some of the factors are assumed to be common to two or more variables, while others are specific to each parameter. Characteristic (unique) factors are orthogonal to one another, that is, they do not contribute to the covariance between the variables. In other words, only common factors that are much smaller than the number of variables contribute to the covariance between them. The latent factor structure can be accurately identified by examining the resulting covariance matrix. In practice, it is impossible to obtain the exact structure of the factor model, only estimates of the parameters of the factor structure can be found. Therefore, on the principle of postulate of parsimony, adopt a model with a minimum number of common factors.

One of the methods of factor analysis is the analysis of principal components. Principal components (PCs) are linear combinations of observed variables that have orthogonality properties, that is, natural orthogonal functions. Thus, PCs are opposite to common factors, since the latter are hypothetical and are not expressed through a combination of variables, whereas PCs are linear functions of the observed variables.

The essence of the PCs method lies in the linear transformation and condensation of the original information. On the basis of correlation matrices, a system of orthogonal, linearly independent functions, nominated by eigenvectors, corresponding to a system of independent random variables nominated by eigenvalues of the correlation matrix $(\lambda)$ is determined. The first few eigenvalues of the correlation matrix exhaust the bulk of the total field variance, so special attention is given to the first eigenvalues and their corresponding components when analyzing the decomposition results. And since large-scale processes, which are functional systems of the body, are characterized

(C) Zavidnyuk Y.V., Mel'nyk O.I., Mysakovets' O.G., 2019 


\section{by a large variance, it is fair to assume that they are reflected in the first components.}

PCs analysis is a method of converting a given sequence of observed variables into another sequence of variables. The method of obtaining the directions of the principal axes is based on finding the eigenvalues and vectors of correlations (covariates). The eigenvalue $(\lambda)$ is the most important characteristic of the matrix; it is used in the decomposition of the covariance matrix and at the same time as a criterion for determining the number of distinguished factors and as a measure of the variance corresponding to this factor. The eigenvector is a vector associated with the corresponding eigenvalue and obtained in the process of isolation of the primary factors. These vectors, presented in the normalized form, are factor loadings.

The first eigenvalue represents the magnitude of the variance corresponding to the first principal axis, the second to the second, etc. The sum of the eigenvalues is equal to the number of variables, and the proportion of variance corresponding to this direction or PC is obtained by dividing the eigenvalue by the number of variables. The task of the PCs is to explain the maximum proportion of the variance of observations, while the task of common factors is to explain the correlations between the variables.

In the $n$-dimensional factor space, the first PC is the representation of points (data) along the selected principal axis, it reproduces the maximum fraction of variance of the experimental data. If each point in the new coordinate system is described, then no information is lost. In the case of a linear relationship between the variables, the first PC contains all the information to describe each point, but if the variables are independent, the principal axis is missing, and the PC analysis does not contribute to even minimal compression of the observation results. In the presence of a more or less close relationship between the variables, the rest of the information is contained in the following PCs, with the axis of the second PC perpendicular to the axis of the first PC and along it a smaller part of the data is located, that is, the second PC reproduces the next largest fraction of variance; even less information is contained along the axis of the third PC, perpendicular to the first two, etc.

A factor structure is considered the simplest if all variables have a single factor complexity, that is, when each variable has a non-zero load on only one common factor. If there are at least two factors, then each row contains only one non-zero element, each column has several zeros, for each pair of columns the zero elements do not match. However, such a simple structure for real data is unattainable. The simplicity of the structure is determined if there are at least three variables for each factor that have a significant load on that factor.

Primary factor loadings are projections of variables on the axis of the n-dimensional space ( $\mathrm{n}$ is the number of factors), that is, the loads are determined by lowering the perpendicular from a given point to the primary orthogonal axes. A simple factor structure is obtained when all the values of the variables lie on these axes. In the orthogonal case, a simple structure is given by a set of points that have non-zero loadings on only one factor (axis). The projection is non-zero if the angle between the cluster of points is different from the straight line. Therefore, we can assume that the accumulation of points is on the primary axes, or that the projections of points on the secondary axes are zero.

The concept of oblique (non-orthogonal) factors is used to simplify the interpretation of the solutions, which makes it possible to better represent the clusters of variables without abandoning the orthogonality (independence) of the factors. Therefore, after determining the clusters of variables and the rotation of the axes within the clusters, the correlation between the found oblique factors is calculated.

In order to find the factor mapping matrix closest to the simplest ideal structure, an orthogonal rotation procedure is performed using quartimax, varimax, and equamax methods. Varimax is a method of obtaining an orthogonal 
solution that reduces to a factor structure using the criterion for minimizing the column of the factor mapping matrix; quartimax is a criterion for obtaining an orthogonal solution, which is reduced to simplifying the description of the rows of the matrix, and equamax combines the properties of the first two.

At the same time, factor analysis is used as a heuristic method of allocating clusters among the registered data, since the found structures are considered as hypotheses, reflecting in the obtained data some tendencies for clustering of variables into clusters.

Now let's demonstrate the practical application of factor analysis.

Material and methods. The study subjects were 58 healthy female Wistar rats 240-290 g, 10 of which were intact and the others were subjected to water-salt loading for 6 days [2,3].

The day after the completion of the drinking course in all rats, at first, a sample of peripheral blood (by incision of the tip of the tail) was taken for leukocytogram analysis. Then they assessed the state of autonomous regulation. For this purpose, under an easy ether anesthesia, for 15-20 sec ECG was recorded in the lead II, inserting needle electrodes under the skin of the legs, followed by the calculation of the parameters of the HRV: moda (Mo), amplitude of the moda (AMo) and variational swing (MxDMn) as markers of the humoral channel of regulation, sympathetic and vagal tones respectively [4].

Animals were then placed in individual chambers with perforated bottom for collecting daily urine. The experiment was completed by decapitation of rats in order to collect as much blood as possible.

The plasma levels of the hormones of adaptation were determined: corticosterone, triiodothyronine and testosterone (by the ELISA [5]); as well as electrolytes: calcium (by reaction with arsenase III), magnesium (by reaction with colgamite), phosphates (phosphate-molybdate method), chloride (mercury-rhodanidine method), sodium and potassium (both in plasma and in erythrocytes) by flamming photometry; nitric metabolites: creatinine (by Jaffe's color reaction by Popper's method), urea (urease method by reaction with phenolhypochlorite), uric acid (uricase method), medium molecular polypeptides (by spectrophotometric method), bilirubin (by diazoreaction using the Jedrashik-Kleghorn-Grof method); lipid peroxidation products: diene conjugates (spectrophotometry of the heptane phase of the lipids extract) and malonic dyaldehide (in the test with thiobarbituric acid), antioxidant enzymes: superoxide dismutase erythrocytes (according to the degree of inhibition of reduction of nitroblue tetrazolium in the presence of $\mathrm{N}$-methylphenazonium metasulphate and $\mathrm{NADH}$ ) and catalase plasma (at the rate of decomposition of hydrogen peroxide), as well as amylase (Karavay's amyloclastic method with starch substrate) and glucose (glucose-oxidase method).

Most of the listed parameters of metabolism were also determined in daily urine. By the size of the diuresis and the level of creatinine in plasma and urine, glomerular filtration and tubular reabsorption were calculated. In addition, the osmolarity of the urine was measured by the cryostatic method.

The analyzes were carried out according to the instructions described in the manual [6]. The analyzers "Tecan" (Oesterreich), "Pointe-180" ("Scientific", USA) and "Reflotron" (Boehringer Mannheim, BRD) were used with appropriate sets and a fiery spectrophotometer "CФ-47".

According to the parameters of electrolyte exchange, hormonal activity was evaluated: parathyroid by coefficients (Cap/Pp) 0,5 and $(\mathrm{Cap} \cdot \mathrm{Pu} / \mathrm{Pp} \cdot \mathrm{Cau}) 0,25$, calcitonin by coefficients $(1 / \mathrm{Cap} \cdot \mathrm{Pp}) 0,25$ and $(\mathrm{Cau} \cdot \mathrm{Pu} / \mathrm{Cap} \cdot \mathrm{Pp}) 0,25$ as well as mineralocorticoid by coefficients $(\mathrm{Nap} / \mathrm{Kp}) 0,5$ and $(\mathrm{Nap} \cdot \mathrm{Ku} / \mathrm{Kp} \cdot \mathrm{Nau}) 0,25$, based on their classical effects and recommendations by IL Popovych $[7,8]$.

In the blood, the parameters of immunity were determined according to the tests of the 1st and 2nd levels of the WHO, as described in the manual [9]: the relative content of the population of T-lymphocytes in a test of spontaneous rosette formation with erythrocytes of sheep by M Jondal et al [10], 
their theophylline-resistant (T-helper) and theophyllin-susceptible (T-cytolytic) subpopulations (by the test of sensitivity of rosette formation to theophylline by S Limatibul et al [11]; the population of B-lymphocytes by the test of complementary rosette formation with erythrocytes of sheep by C Bianco [12]. Natural killers were identified as large granules contain lymphocytes.

About the state of the phagocytic function of neutrophils (microphages) and monocytes (macrophages) were judged by the phagocyte index, the microbial count and the killing index for Staphylococcus aureus (ATCC N25423 F49) $[13,14]$.

After decapitation, the spleen, thymus and adrenal glands were removed from the animals. Immune organs weighed and made smears-imprints for counting splenocytogram and thymocytogram [13]. For them, as well as leukocytogram, Shannon's entropy was calculated $[15,16]$. In the adrenal glands after weighing, the thickness of glomerular, fascicular and reticular zones was measured under a microscope [13].

Factor analysis (principal component method) was implemented with the program Statistica 5.5.

Results and discussion. It is believed that to study the factor structure of the investigated field can be limited to considering the number of PCs, the total contribution of which to the total variance of the original data exceeds 2/3. Another approach for determining the number of PCs is to apply the Kaiser $(\lambda>1)$ and Cattell (with the maximum deceleration of the eigenvalue $\lambda$, graphically visualized) criteria [1].

In the first stage of the analysis, it was found that the dispersion of the information field of 106 parameters of the neuro-endocrine-immune complex and metabolism is absorbed by 20 PCs (Fig. 1). Using the Cattel method, the number of PCs analyzed is limited to twelve, the total contribution of which to the total variance of the raw data is $66,2 \%$ (Table 1 ), ie, it reaches the required critical level.

The summary of factor analysis of the field of variables is shown in Table 2 , which is essentially a matrix of factor mapping whose elements are factor loadings, that is, the correlation coefficients between PC and variables.

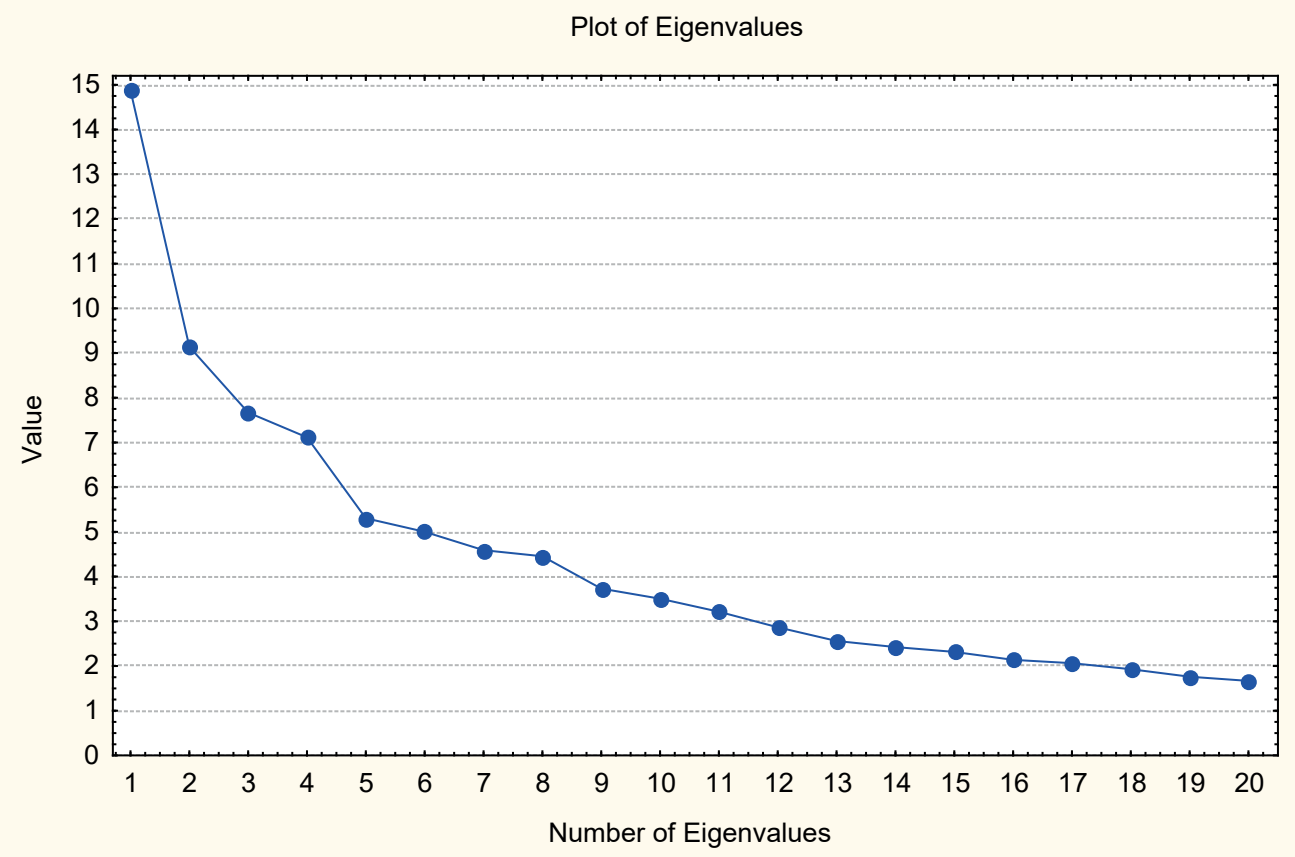

Fig. 1. Eigenvalues of principal components 
Table 1.

Eigenvalues. Extraction: Principal components

\begin{tabular}{|c|c|c|c|c|}
\hline & Eigenval & $\begin{array}{c}\text { \% total } \\
\text { Variance }\end{array}$ & $\begin{array}{c}\text { Cumul } \\
\text { Eigenval }\end{array}$ & $\begin{array}{c}\text { Cumul } \\
\text { \% }\end{array}$ \\
\hline 1 & 14,88 & 13,8 & 14,88 & 13,8 \\
\hline 2 & 9,14 & 8,5 & 24,02 & 22,2 \\
\hline 3 & 7,67 & 7,1 & 31,69 & 29,3 \\
\hline 4 & 7,12 & 6,6 & 38,80 & 35,9 \\
\hline 5 & 5,31 & 4,9 & 44,11 & 40,8 \\
\hline 6 & 5,00 & 4,6 & 49,11 & 45,5 \\
\hline 7 & 4,59 & 4,3 & 53,70 & 49,7 \\
\hline 8 & 4,45 & 4,1 & 58,15 & 53,8 \\
\hline 9 & 3,73 & 3,5 & 61,88 & 57,3 \\
\hline 10 & 3,51 & 3,2 & 65,39 & 60,5 \\
\hline 11 & 3,21 & 3,0 & 68,60 & 63,5 \\
\hline 12 & 2,87 & 2,7 & 71,47 & 66,2 \\
\hline
\end{tabular}

Table 2 .

Factor loadings (Varimax normalized). Clusters of loadings, those determine the oblique factors for hierarchical analysis of parameters of male rats (Marked loadings are $>0,700$

\begin{tabular}{|c|c|c|c|c|c|c|c|c|c|c|c|c|}
\hline & & & & & & & & & & & & \\
\hline & 929 & 009 & 93 & 025 & 003 & 035 & & & 002 &, 026 & & \\
\hline & & & & & & & & & & & & \\
\hline & & & & & & & & & & & & \\
\hline & 385 & 034 & 035 & & & & & & & & & \\
\hline & & & & & & & & & & & & \\
\hline & & & & & & & & & & & & \\
\hline & & & & & & & & & & & & 0 \\
\hline & & & & & & & & & & & & \\
\hline & 693 & & & & & 29 & 023 & & & & & \\
\hline R & & & & & & & & & & & & \\
\hline & & & & & & & & & & & & \\
\hline $\mathrm{N}$ & 13 & $-0,033$ & 116 & $-0,105$ & ,092 & & 102 & & & & & 20 \\
\hline & & & & & & & & & & & & 4 \\
\hline & 102 & & & $-0,276$ & 085 & & & & & & & \\
\hline IU & 345 & 911 & 35 & $-0,016$ & 030 & $-0,045$ & $-0,015$ & & & 0,047 & &, 14 \\
\hline & & & & & & & & & & & & 14 \\
\hline r & & & & & & & & & & & & 05 \\
\hline & & & & & & & & & & & & \\
\hline & & & & & & & & & & & & \\
\hline & & & 996 & & & & 246 & & 289 & $-0,045$ & &, 1 \\
\hline & & & & & & & & & & & & \\
\hline & & & & & & & & & & & &, 0 \\
\hline & & & & & & & & & & & &, 00 \\
\hline & & & & & & & & & & & & \\
\hline LU & $-0,326$ & ,302 & $-0,905$ & $-0,001$ & $-0,045$ & $-0,006$ & $-0,043$ & $-0,149$ & $-0,108$ & 0,100 & 0,016 &, 12 \\
\hline
\end{tabular}




\begin{tabular}{|c|c|c|c|c|c|c|c|c|c|c|c|c|}
\hline ariables & $1 \mathrm{PC}$ & $2 \mathrm{PC}$ & $3 \mathrm{PC}$ & $4 \mathrm{PC}$ & $5 \mathrm{PC}$ & $6 \mathrm{PC}$ & $7 \mathrm{PC}$ & $8 \mathrm{PC}$ & $9 \mathrm{PC}$ & $10 \mathrm{PC}$ & $11 \mathrm{PC}$ & 10 \\
\hline CLEX & $-0,438$ & $-0,313$ & $-0,892$ & $-0,053$ & 0,028 & 0,036 & 0,005 & $-0,009$ & $-0,064$ & 0,075 & $-0,005$ & 0,131 \\
\hline TAEX & 0,374 & $-0,073$ & $-0,815$ & $-0,018$ & 0,049 & 0,040 & $-0,094$ & 0,248 & 0,068 & $-0,057$ & $-0,018$ & $-0,009$ \\
\hline ICA4 & 0,159 & 0,403 & 0,807 & 0,159 & $-0,096$ & $-0,065$ & 0,126 & $-0,333$ & $-0,009$ & 0,029 & 0,026 & 0,140 \\
\hline ICAU & ,158 & 0,398 & 0,777 & 0,161 &, 015 & $-0,097$ & 0,152 & $-0,246$ & $-0,014$ & 0,090 & $-0,016$ & 0,140 \\
\hline LUC & 0,158 & 0,342 & 0,415 & $-0,055$ & 0,149 & $-0,080$ & $-0,296$ & $-0,122$ & 244 & 096 & 092 &, 143 \\
\hline CRP & 0,157 & 0,289 & 0,293 & 0,829 & 0,156 & $-0,144$ & 0,079 & 0,168 & $-0,062$ & $-0,042$ & 0,052 & ,179 \\
\hline UREA & 0,144 & 0,267 & 0,283 & 0,748 & 0,030 & 0,075 & 0,199 & $-0,055$ & $-0,074$ & $-0,253$ & 0,000 & $-0,149$ \\
\hline & 177 & 0,536 & $-0,037$ & $-0,575$ & $-0,013$ & 0,073 & $-0,108$ & & & & & 181 \\
\hline CORT_2 & 0,136 & 0,216 & 0,233 & 0,416 & 0,128 & $-0,050$ & 0,199 & 0,204 & $-0,119$ & 0,281 & $-0,188$ & $-0,101$ \\
\hline PL\% & 0,130 & 0,214 &, 198 & 0,405 & 0,036 & 0,180 & $-0,001$ & $-0,359$ & 0,095 & 229 & 325 & 289 \\
\hline $\mathrm{F}$ & 0,136 & 0,220 & 0,235 & 0,435 & 0,879 & 0,011 & 0,032 & 0,033 & 0,068 & $-0,086$ & 175 &, 008 \\
\hline $\mathrm{EG}$ & $-0,249$ & $-0,217$ & $-0,261$ & $-0,345$ & $-0,851$ & $-0,068$ & $-0,060$ & $-0,027$ & $-0,047$ & $-0,005$ & $-0,153$ & 0,042 \\
\hline PAL & $-0,258$ & $-0,223$ & $-0,333$ & $-0,349$ & $-0,741$ & $-0,002$ & 0,047 & 0,225 & $-0,045$ & 0,144 & $-0,088$ & $-0,085$ \\
\hline ET & 126 & 0,202 & 0,197 & 0,396 & 0,378 & $-0,145$ & & $-0,075$ & & & & 186 \\
\hline n & ,267 & $-0,280$ & $-0,346$ & $-0,620$ & $-0,349$ & 0,137 & 0,169 & $-0,034$ & 0,075 & 0,084 & 0,250 & $-0,037$ \\
\hline TA4 & 0,126 & 0,192 & 0,175 & 0,342 & 0,377 & 0,758 & 0,031 & 0,146 & 0,070 & 0,052 & 0,109 & 0,335 \\
\hline TAP & 0,126 & 0,179 & 0,153 & 0,261 & 0,309 & 0,747 & 0,070 & 0,240 & 0,043 & 0,009 & 0,048 & 0,017 \\
\hline CTA4 & 0,120 & 0,134 & 0,142 & 0,254 & 0,279 & 0,738 & $-0,048$ & 0,234 & $-0,058$ & 0,067 & $-0,066$ & $-0,253$ \\
\hline CTAP & 0,105 & 0,123 & 0,137 & 0,242 & 0,259 & 0,661 & $-0,049$ & 0,077 & $-0,129$ & 0,206 & $-0,057$ & 0,251 \\
\hline PU & 0,143 & 0,226 & 0,236 & 0,451 & 0,002 & 0,540 & 0,045 & 0,261 & 0,157 & $-0,059$ & 0,221 & $-0,004$ \\
\hline MGP & ,235 & $-0,208$ & $-0,248$ & $-0,303$ & $-0,317$ & $-0,485$ & $-0,189$ & 0,333 & 0,194 & 0,070 & 0,074 & $-0,125$ \\
\hline EUS & 0,101 & 0,123 & 0,131 & 0,232 & 0,257 & 0,476 & $-0,207$ & $-0,209$ & 0,247 & 0,025 & 011 & $-0,016$ \\
\hline IDA &, 235 & $-0,206$ & ,232 & $-0,282$ & $-0,261$ & $-0,475$ & $-0,225$ & 0,158 & $-0,040$ & $-0,080$ & $-0,146$ & 0,142 \\
\hline CHOL & 0,099 & 0,109 & 0,124 & 0,222 & 0,216 & 0,374 & $-0,004$ & 0,352 & 0,285 & $-0,153$ & 051 & $-0,045$ \\
\hline TEST & $-0,181$ & $-0,129$ & $-0,182$ & $-0,198$ & $-0,156$ & $-0,329$ & 0,190 & $-0,107$ & 0,038 & $-0,274$ & $-0,121$ & $-0,127$ \\
\hline AMO & 0,079 & 0,101 & 0,099 & 0,199 & 0,193 & 0,183 & 0,789 & $-0,222$ & 0,018 & $-0,131$ & 058 & 0,068 \\
\hline MO & $-0,189$ & $-0,154$ & $-0,183$ & $-0,228$ & $-0,171$ & $-0,334$ & $-0,756$ & 0,225 & $-0,009$ & 0,128 & & $-0,017$ \\
\hline DX & $-0,193$ & $-0,168$ & $-0,190$ & $-0,255$ & $-0,184$ & $-0,359$ & $-0,753$ & 0,101 & 0,144 & 0,015 & 0,077 & $-0,046$ \\
\hline $\mathrm{C}$ & $-0,197$ & $-0,173$ & $-0,195$ & $-0,276$ & $-0,213$ & $-0,373$ & $-0,687$ & 0,003 & 0,157 & $-0,051$ & $-0,173$ & 0,040 \\
\hline IAP & ,076 & 0,101 & 0,083 & 0,188 & 0,181 & 0,177 & 0,548 & 0,134 & 0,164 & 0,121 & 0,052 & 0,229 \\
\hline $\mathrm{CU}$ & $-0,209$ & $-0,184$ & $-0,210$ & $-0,278$ & $-0,221$ & $-0,411$ & $-0,477$ & $-0,098$ & $-0,035$ & 0,011 & 0,075 & 0,079 \\
\hline CLP & 0,068 & 0,100 & 0,080 & 0,181 & 0,176 & 0,161 & 0,458 & 0,183 & 0,167 & 0,297 &, 017 & 0,165 \\
\hline $\mathrm{SC}$ & 0,058 & 0,080 & 0,079 & 0,176 & 0,170 & 0,153 & 0,454 & $-0,438$ & 0,047 & $-0,091$ & & $-0,034$ \\
\hline PLAS & & 0,107 & & & & & & & & & & $-0,255$ \\
\hline CRU &, 140 & $-0,079$ & $-0,123$ & $-0,125$ & $-0,100$ & $-0,153$ & $-0,080$ & $-0,759$ & 0,044 & $-0,035$ & $-0,049$ & 141 \\
\hline MMMU & 0,054 & 0,078 & 0,078 & 0,158 & 0,166 & 0,151 & 0,363 & 0,651 & 0,018 & $-0,002$ & $-0,092$ & 0,107 \\
\hline CREAB & $-0,145$ & $-0,092$ & $-0,130$ & $-0,135$ & $-0,108$ & $-0,187$ & $-0,082$ & $-0,643$ & 0,047 & $-0,069$ & $-0,064$ & 0,069 \\
\hline EPYT & $-0,148$ & $-0,093$ & $-0,144$ & $-0,146$ & $-0,127$ & $-0,191$ & $-0,095$ & $-0,620$ & 0,028 & $-0,084$ & 0,053 & $-0,096$ \\
\hline JAER & 0,050 & 0,074 & 0,077 & 0,153 & 0,161 & 0,149 & 0,301 & 0,600 & 0,076 & 0,099 & 0,173 & $-0,149$ \\
\hline $\mathrm{KU}$ & $-0,152$ & $-0,093$ & $-0,152$ & $-0,151$ & $-0,136$ & $-0,195$ & $-0,113$ & $-0,596$ & $-0,294$ & 0,179 & 0,057 & 0,342 \\
\hline LBS & 0,045 & 0,069 & 0,074 & 0,142 & 0,148 & 0,145 & 0,271 & 0,566 & 0,070 & $-0,211$ & 0,014 & 0,034 \\
\hline KAT & 0,042 & 0,068 & 0,064 & 0,140 & 0,138 & 0,140 & 0,250 & 0,495 & $-0,080$ & $-0,047$ & 0,212 & $-0,218$ \\
\hline BIL & 0,027 & 0,059 & 0,062 & 0,136 & 0,120 & 0,127 & 0,231 & 0,466 & $-0,121$ & $-0,122$ & 0,032 & $-0,124$ \\
\hline MMM & 0,026 & 0,053 & 0,055 & 0,126 & 0,090 & 0,095 & 0,213 & 0,432 & $-0,089$ & 0,191 & $-0,114$ & $-0,021$ \\
\hline LBT & 0,021 & 0,053 & 0,051 & 0,123 & 0,082 & 0,086 & 0,194 & 0,420 & $-0,037$ & 0,310 & 0,169 & $-0,071$ \\
\hline AMYL & $-0,161$ & $-0,095$ & $-0,153$ & $-0,153$ & $-0,141$ & $-0,197$ & $-0,117$ & $-0,470$ & 0,058 & $-0,035$ & 0,153 & $-0,018$ \\
\hline IIDAU & $-0,162$ & $-0,096$ & $-0,161$ & $-0,154$ & $-0,146$ & $-0,219$ & $-0,119$ & $-0,438$ & 0,056 & $-0,098$ & $-0,008$ & 0,067 \\
\hline KER & $-0,165$ & $-0,108$ & $-0,178$ & $-0,157$ & $-0,151$ & $-0,234$ & $-0,170$ & $-0,437$ & 0,184 & 0,255 & 0,271 & 0,039 \\
\hline
\end{tabular}




\begin{tabular}{|c|c|c|c|c|c|c|c|c|c|c|c|c|}
\hline Variables & $1 \mathrm{PC}$ & $2 \mathrm{PC}$ & $3 \mathrm{PC}$ & $4 \mathrm{PC}$ & $5 \mathrm{PC}$ & $6 \mathrm{PC}$ & $7 \mathrm{PC}$ & $8 \mathrm{PC}$ & $9 \mathrm{PC}$ & $10 \mathrm{PC}$ & $11 \mathrm{PC}$ & $12 \mathrm{PC}$ \\
\hline AMYU & $-0,179$ & $-0,116$ & $-0,178$ & $-0,195$ & $-0,155$ & $-0,272$ & $-0,230$ & $-0,419$ & 0,396 & 0,031 & $-0,033$ & $-0,151$ \\
\hline ETT & $-0,182$ & $-0,146$ & $-0,182$ & 0,216 & $-0,158$ & $-0,333$ & 0,268 & $-0,367$ & 0,174 & $-0,040$ & 0,113 & $-0,193$ \\
\hline GAST & $-0,111$ & $-0,071$ & $-0,079$ & $-0,087$ & $-0,076$ & $-0,090$ & $-0,059$ & $-0,184$ &, 628 & $-0,304$ & 0,042 & $-0,193$ \\
\hline RETS & $-0,112$ & $-0,077$ & $-0,087$ & $-0,095$ & $-0,087$ & $-0,115$ & $-0,063$ & $-0,216$ & $-0,625$ & 0,074 & 0,055 & 0,035 \\
\hline ТHYM\% & 0,021 & 0,052 & 0,047 & 0,118 & 0,081 & 0,085 & 0,191 & 0,356 & 0,598 & $-0,082$ & 0,079 & 0,170 \\
\hline MASA & $-0,121$ & $-0,077$ & $-0,094$ & $-0,109$ & $-0,095$ & $-0,143$ & $-0,067$ & $-0,286$ & $-0,558$ & $-0,094$ & $-0,020$ & 0,058 \\
\hline GLO & 0,019 & 0,050 & 0,036 & 0,115 & 0,078 & 0,084 & 0,188 & 78 & 0,534 & 0,064 & 175 & 106 \\
\hline LEU & 0,013 & 0,046 & 0,034 & 0,112 & 0,077 & 0,066 & 0,161 & 0,270 & 0,467 & $-0,210$ & $-0,040$ & 0,070 \\
\hline LFS & 0,013 & 0,042 & 0,029 & 0,099 & 0,062 & 0,044 & 0,155 & 0,242 & 0,443 & 0,054 & 0,262 & 0,242 \\
\hline FIBS & $-0,125$ & $-0,078$ & $-0,114$ & $-0,110$ & $-0,099$ & $-0,151$ & $-0,074$ & $-0,346$ & $-0,358$ & 0,030 & $-0,264$ & 0,067 \\
\hline $\mathrm{HT}$ & $-0,086$ & $-0,056$ & $-0,051$ & $-0,037$ & $-0,049$ & $-0,032$ & $-0,035$ & $-0,144$ & $-0,206$ & $-0,686$ & & $-0,235$ \\
\hline LCT & 0,026 & 0,059 & 0,061 & 0,134 & 0,100 & 0,096 & 0,227 & 0,442 & 0,095 & 0,558 & $-0,143$ & 0,258 \\
\hline PLAT & $-0,086$ & $-0,056$ & $-0,052$ & $-0,048$ & $-0,055$ & $-0,059$ & $-0,039$ & $-0,158$ & $-0,213$ & $-0,544$ & 0,091 & $-0,108$ \\
\hline ENDT & $-0,095$ & $-0,061$ & $-0,054$ & $-0,068$ & $-0,061$ & $-0,076$ & $-0,058$ & $-0,163$ & $-0,215$ & $-0,469$ & $-0,311$ & 38 \\
\hline MACT & $-0,107$ & $-0,070$ & $-0,066$ & $-0,072$ & $-0,072$ & $-0,088$ & $-0,059$ & $-0,176$ & $-0,330$ & $-0,441$ & 0,135 & $-0,103$ \\
\hline CAP & 0,010 & 0,041 & 0,025 & 0,097 & 0,061 & 0,040 & 0,151 & 0,173 & 0,268 & 0,402 & $-0,065$ & 0,064 \\
\hline $\mathrm{KP}$ & 0,006 & 0,027 & 0,023 & 0,094 & 0,058 & 0,035 & 0,139 & 0,166 & 0,214 & 0,391 & $-0,232$ & $-0,053$ \\
\hline MCAP & $-0,112$ & $-0,075$ & $-0,084$ & $-0,088$ & $-0,086$ & $-0,097$ & $-0,059$ & $-0,192$ & $-0,450$ & $-0,374$ & 0,307 & 0,086 \\
\hline EO & $-0,013$ & 0,022 & 0,015 & 0,093 & 0,047 & 0,031 & 0,114 & 0,131 & 0,117 & 0,351 & $-0,025$ & $-0,201$ \\
\hline $\mathrm{O}$ & $-0,085$ & $-0,054$ & $-0,050$ & $-0,030$ & $-0,044$ & $-0,027$ & $-0,026$ & $-0,119$ & $-0,138$ & $-0,130$ & $-0,876$ & 0,115 \\
\hline B & $-0,019$ & 0,013 & 0,014 & 0,092 & 0,046 & 0,031 & 0,093 & 0,116 & 0,115 & 0,313 & 0,753 & 0,022 \\
\hline TS & $-0,025$ & 0,001 & 0,011 & 0,067 & 0,045 & 0,027 & 0,087 & 0,110 & 0,095 & 0,184 & 0,652 & 0,191 \\
\hline $\mathrm{TH}$ & $-0,038$ & $-0,003$ & 0,007 & 0,066 & 0,037 & 0,022 & 0,081 & 0,101 & 0,083 & 0,104 & 0,530 & $-0,490$ \\
\hline SOD & $-0,040$ & $-0,008$ & 0,003 & 0,055 & 0,020 & 0,021 & 0,080 & 0,077 & 0,071 & 0,074 & 0,421 & $-0,153$ \\
\hline BAS & $-0,078$ & $-0,049$ & $-0,040$ & $-0,030$ & $-0,039$ & $-0,018$ & $-0,024$ & $-0,110$ & $-0,129$ & $-0,125$ & $-0,329$ & $-0,034$ \\
\hline $\mathrm{ADR} \%$ & $-0,064$ & $-0,038$ & $-0,034$ & $-0,013$ & $-0,027$ & $-0,007$ & 0,013 & $-0,064$ & $-0,077$ & $-0,077$ & $-0,244$ & $-0,174$ \\
\hline PTAU & $-0,046$ & $-0,012$ & $-0,002$ & 0,050 & 0,018 & 0,016 & 0,079 & 0,054 & 0,040 & 0,049 & 0,182 & 0,730 \\
\hline $\mathrm{CAU}$ & $-0,047$ & $-0,022$ & $-0,008$ & 0,046 & 0,017 & 0,013 & 0,068 & 0,045 & 0,035 & 0,033 & 0,008 & $-0,639$ \\
\hline HI & $-0,063$ & $-0,031$ & $-0,029$ & 0,000 & $-0,023$ & 0,004 & 0,025 & 0,019 & $-0,044$ & $-0,067$ & $-0,171$ & 0,597 \\
\hline ES & $-0,053$ & $-0,024$ & $-0,017$ & 0,031 & 0,011 & 0,011 & 0,068 & 0,025 & 0,019 & $-0,014$ & $-0,049$ & $-0,487$ \\
\hline CTAU & $-0,012$ & 0,025 & 0,020 & 0,093 & 0,057 & 0,033 & 0,132 & 0,136 & 0,199 & 0,387 & $-0,035$ & $-0,464$ \\
\hline HS & $-0,072$ & $-0,047$ & $-0,034$ & $-0,017$ & $-0,029$ & $-0,012$ & 0,004 & $-0,087$ & $-0,085$ & $-0,080$ & $-0,287$ & $-0,458$ \\
\hline FIM & $-0,055$ & $-0,025$ & $-0,021$ & 0,025 & $-0,019$ & 0,008 & 0,039 & 0,021 & $-0,001$ & $-0,019$ & $-0,122$ & 0,322 \\
\hline $\begin{array}{l}\text { Expl. } \\
\text { Var }\end{array}$ & 10,42 & 8,03 & 7,17 & 5,27 & 4,57 & 5,32 & 5,02 & 8,60 & 4,41 & 3,77 & 4,03 & 4,84 \\
\hline Prp. & 0,097 & 0,074 & 0,066 & 0,049 & 0,042 & 0,049 & 0,047 & $\mathbf{0 , 0 8}$ & 0,041 & 0,035 & 0,037 & 0,045 \\
\hline
\end{tabular}

In the second step, we obtained a correlation matrix for oblique factors, which was subjected to further analysis to distinguish a plurality of orthogonal factors that divide the variability into variables related to total variance (secondary factors) and to individual variations or cluster-related variables (primary factors).

It is revealed (Table 3 ) that there are 3 directly unmeasured hypothetical common factors. They combines, on the one hand, the immune and neuro-endocrine parameters, and on the other hand, there are elements of the metabolism. 
Table 3 .

Secondary (S) Factors Loadings at female rats

\begin{tabular}{|c|c|c|c|}
\hline & S 1 & S 2 & S 3 \\
\hline MACS &,- 55 &, 04 & ,06 \\
\hline LBS &, 55 & ,04 & ,02 \\
\hline PLAS &, 53 &,- 00 &, 27 \\
\hline EPYT &,- 53 &,- 28 &,- 12 \\
\hline $\mathrm{AMO}$ &,- 52 & ,01 &,- 03 \\
\hline $\mathrm{MO}$ & ,52 &,- 08 &,- 03 \\
\hline NAER & , 52 & ,01 &, 16 \\
\hline CRU &,- 51 &,- 13 &,- 05 \\
\hline NAEX & 48 &, 10 &,- 03 \\
\hline T3 &,- 48 &, 12 &,- 24 \\
\hline MCA4 &,- 48 & ,06 & ,03 \\
\hline FNN &, 47 &,- 13 & ,19 \\
\hline LCT & ,46 & ,36 &,- 05 \\
\hline $\mathrm{DX}$ &, 45 &,- 21 &,- 04 \\
\hline AMYL &,- 45 &,- 10 &, 13 \\
\hline NK &,- 42 &, 15 &,- 24 \\
\hline MON &,- 42 &, 13 &,- 17 \\
\hline KU &,- 41 &,- 05 &,- 17 \\
\hline $\mathrm{MCAU}$ &,- 40 &, 08 &, 06 \\
\hline HT &,- 40 &,- 32 & ,09 \\
\hline NAU & 38 &,- 09 &,- 07 \\
\hline KAT & ,38 & 20 & ,36 \\
\hline UE\% & ,37 & ,38 & 06 \\
\hline KATU & ,36 & ,26 &, 27 \\
\hline MGU &,- 36 &,- 04 & ,11 \\
\hline MMMU &, 35 &, 23 &, 02 \\
\hline $\mathrm{PP}$ &,- 34 &, 07 &,- 11 \\
\hline CAE\% & ,33 & ,33 & ,36 \\
\hline CTA4 & ,33 &,- 02 &, 17 \\
\hline MDAU &,- 32 &,- 21 &,- 01 \\
\hline CHOL & 32 &, 00 & ,03 \\
\hline UREAU & 31 &, 17 & ,09 \\
\hline CLEX & ,30 &, 11 &,- 16 \\
\hline FIN & 30 &,- 22 &, 17 \\
\hline GAST &,- 30 &, 15 & ,27 \\
\hline TEST &,- 28 &, 01 & ,09 \\
\hline $\mathrm{LF}$ &, 15 &,- 47 & ,16 \\
\hline $\mathrm{HL}$ &,- 33 &, 45 &,- 17 \\
\hline DIU\% & 25 &, 44 & ,00 \\
\hline
\end{tabular}




\begin{tabular}{|c|c|c|c|}
\hline & S 1 & S 2 & S 3 \\
\hline PE\% &, 34 &, 41 &, 07 \\
\hline PAL &,- 01 &, 40 &,- 04 \\
\hline SEG &,- 05 &, 38 &,- 12 \\
\hline AMYU &,- 33 &,- 37 &, 07 \\
\hline O &, 00 &, 36 &,- 10 \\
\hline CREX &,- 05 &, 35 &,- 04 \\
\hline KE\% &,- 09 &, 34 &,- 11 \\
\hline THYM\% &,- 18 &,- 34 &,- 11 \\
\hline URU &, 10 &,- 33 &,- 05 \\
\hline B &, 16 &,- 33 &, 07 \\
\hline NEUS &, 01 &,- 32 &,- 01 \\
\hline FIBS &,- 16 &, 30 &, 07 \\
\hline TS &, 04 &,- 29 &,- 14 \\
\hline CRP &,- 06 &, 06 &, 59 \\
\hline CAU &, 24 &, 03 &, 58 \\
\hline PTAU &,- 03 &,- 02 &,- 53 \\
\hline UREA &,- 22 &, 09 &, 49 \\
\hline CREAB &,- 33 &,- 10 &,- 46 \\
\hline GF100 &, 11 &, 20 &,- 43 \\
\hline HS &, 10 &, 21 &, 41 \\
\hline TH &, 08 &,- 27 &, 39 \\
\hline CTAU &, 30 &,- 09 &, 37 \\
\hline LFS &, 14 &,- 19 &,- 35 \\
\hline SPL\% &,- 21 &, 12 &, 32 \\
\hline PTA4 &, 25 &,- 02 &,- 31 \\
\hline CORT_2 &, 00 &, 20 &, 29 \\
\hline & & & \\
\hline
\end{tabular}

Therefore, due to factor analysis, 3 independent clusters of parameters of the neuro-endocrine-immune complex and metabolism, related to cause/effect functional relationships, were identified.

You can see that there is a general pattern - the combination of neuro-endocrine and immune parameters in 8 individual PCs, which, in our material, illustrates the correctness of the concept of a triple neuroendocrine-immune complex $[17,18]$, the elements of which carry out bilateral interaction $[8,19-28]$.

On the other hand, in $10 \mathrm{PCs}$, neuro-endocrine and/or immune parameters are combined with metabolism parameters. The cause/effect relationships between them will be the subject of a subsequent study.

Рекомендовано до друку колісією з біоетики

\section{REFERENCES (ПОСИЛАННЯ)}

1. Kim JO, Mueller Ch W. Factor analysis: statistical methods and practical issues [trans. from English in Russian] (elevent printing, 1986). In: Factor, Discriminant and Cluster Analysis. Moskwa: Finansy i Statistika; 1989: 5-77.

2. Zavidnyuk YV, Mysula IR, Klishch IM, Zukow W, Popovych IL, Korda MM. General non-specific metabolic, neuroendocrine and immune reactions to various water-salt loads in female rats. Journal of Education, Health and Sport. 2018;8(3):513-24.

3. Zavidnyuk YV. Features of metabolic reactions to various water-salt loads in female rats. Experimental and Clinical Physiology and Biochemistry. 2018;2(82):21-30. 
4. Baevskiy RM, Kirillov OI, Kletskin SZ. Mathematical Analysis of Changes in Heart Rate by Stress [in Russian]. Moskva: Nauka; 1984: 221 p.

5. Instructions for the use of a set of reagents for the enzyme-linked immunosorbent assay in human serum. St. Petersburg: Alkor Bio CJSC; 2000: 50 p.

6. Goryachkovskiy AM. Clinical Biochemistry [in Russian]. Odesa: Astroprint; 1998: 608 p.

7. Popovych IL. Factor and canonical analysis parameters of neuro-endocrine-immune complex, metabolism and erosive and ulcerative injuries of stomach mucosa in rats under acute water-immersion stress [in Ukrainian]. Medical Hydrology and Rehabilitation. 2007;5(2):68-80.

8. Popovych IL. Functional interactions between neuroendocrine-immune complex in males rats [in Ukrainian]. Achievements of Clinical and Experimental Medicine. 2008;2(9):80-7.

9. Lapovets' LYe, Lutsyk BD. Handbook of Laboratory Immunology [in Ukrainian]. Lviv; 2002: 173 p.

10. Jondal M, Holm G, Wigzell H. Surface markers on human T and B lymphocytes. I. A large population of lymphocytes forming nonimmune rosettes with sheep red blood cells. J Exp Med. 1972;136(2):207-15.

11. Limatibul S, Shore A, Dosch HM, Gelfand EW. Theophylline modulation of E-rosette formation: an indicator of T-cell maturation. Clin Exp Immunol. 1978;33(3):503-13.

12. Bianco C. Population of lymphocytes bearing a membrane receptor for antigen-antibody complex. J Exp Med. 1970; 134(4): 702-20.

13. Bilas VR, Popovych IL. Role of microflora and organic substances of water Naftussya in its modulating influence on neuroendocrine-immune complex and metabolism [in Ukrainian]. Medical Hydrology and Rehabilitation. 2009;7(1):68-102.

14. Douglas SD, Quie PG. Investigation of Phagocytes in Disease. Churchil; 1981: $110 \mathrm{p}$.

15. Shannon CE. Works on the theory of informatics and cybernetics [transl. from English to Russian]. Moskwa: Inostrannaya literatura; 1963: $329 \mathrm{p}$.

16. Yushkovs'ka OG. Using information theory to study adaptive responses in the body athletes [in Ukrainian]. Medical Rehabilitation, Kurortology, Physiotherapy. 2001;1(25):40-3.

17. Korneva EA, Shkhinek EK, Frolov BA. Neuroendocrine mechanisms of regulation of immune system functions. In: Immunophysiology / Ed Korneva EA. St-Pb: Nauka; 1993: 5-15.

18. Popovych IL. The concept of neuroendocrine-immune complex (Review) [in Russian]. Medical Hydrology and Rehabilitation. 2009;7(3):9-18.

19. Khaitov RM. Physiology of Immune System [in Russian]. Moskva: VINITI RAN; 2005 : $428 \mathrm{p}$

20. Sternberg EM. Neural regulation of innate immunity: a coordinated nonspecific host response to pathogens. Nat Rev Immunol. 2006;6(4):318-28.

21. Uchakin PN, Uchakina ON, Tobin BV, Ershov FI. Neuroendocrine immunomodulation [in Russian]. Vestnik Ross AMN. 2007;9:26-32.

22. Tracey KJ. Reflex control of immunity. Nat Rev Immunol. 2009;9(6):418-28.

23. Thayer JF, Sternberg EM. Neural aspects of immunomodulation: Focus on the vagus nerve. Brain Behav Immun. 2010;24(8):1223-8.

24. Polovynko IS, Zayats LM, Zukow W, Popovych IL. Neuro-endocrine-immune relationships by chronic stress at male rats. Journal of Health Sciences. 2013;3(12):365-74.

25. Zajats LM, Polovynko IS, Zukow W, Yanchij RI, Mysakovets' OG, Mel'nyk OI, Hrytsak YaL. Neuroendocrine-immune relatioships in rats females. Journal of Education, Health and Sport. 2017;7(10):59-78.

26. Chavan SS, Pavlov VA, Tracey KJ. Mechanism and therapeutic relevance of neuro-imune communication. Immunity. 2017;46:927-42.

27. Chavan SS, Tracey KJ. Essencial Neuroscience in Immunology. J Immunol. 2017;198:3389-97.

28. Pavlov VA, Chavan SS, Tracey KJ. Molecular and functional neuroscience in immunity. Annu Rev Immunol. 2018;36:783-812. 


\title{
RESEARCH ARTICLE \\ Factor analysis of the information field of the neuroendocrine- immune complex and metabolism in female rats
}

Y.V. ZAVIDNYUK ${ }^{1,2}$, O.I. MEL'NYK ${ }^{3}$, O.G. MYSAKOVETS' ${ }^{3}$

\author{
${ }^{1} I$.Y. Horbachevs'kyi National Medical University, Ternopil', Ukraine \\ zavidnyukyv@tdmu.edu.ua
}

\author{
${ }^{2}$ Ukrainian Scientific Research Institute of Medicine for Transport, Odesa, Ukraine \\ 3Danylo Halyts'ky National Medical University, L'viv, Ukraine

\section{oksana@meduniv.lviv.ua}

Introduction. Despite considerable informativeness, factor analysis in biomedical research is still rarely used. Therefore, we set out to introduce our colleagues to the theoretical foundations of factor analysis and to demonstrate its application in our own material.

Material and methods. The study subjects were 58 healthy female Wistar rats 240-290 g, 10 of which were intact and the others were subjected to water-salt loading for 6 days.The day after the completion of the drinking course in all rats, at first, a sample of peripheral blood was taken for leukocytogram analysis. Then they assessed the state of autonomous regulation by the parameters of the HRV. Animals were then placed in individual chambers for collecting daily urine. The experiment was completed by decapitation of rats in order to collect as much blood as possible. The plasma levels of the hormones of adaptation were determined: corticosterone, triiodothyronine and testosterone as well as electrolytes: calcium, magnesium, phosphates, chloride, sodium and potassium (both in plasma and in erythrocytes); nitric metabolites: creatinine, urea, uric acid, medium molecular polypeptides, bilirubin; lipid peroxidation products: diene conjugates and malonic dyaldehide, antioxidant enzymes: superoxide dismutase erythrocytes and catalase plasma, as well as amylase and glucose. Most of the listed parameters of metabolism were also determined in daily urine. In addition, the osmolarity of the urine was measured. According to the parameters of electrolyte exchange, hormonal activity was evaluated: parathyroid, calcitonin and mineralocorticoid.In the blood, the parameters of immunity were determined according to the tests of the 1st and 2nd levels of the WHO. The spleen, thymus and adrenal glands were removed. Immune organs weighed and made smears-imprints for counting splenocytogram and thymocytogram. For them, as well as leukocytogram, Shannon's entropy was calculated. In the adrenal glands after weighing, the thickness of glomerular, fascicular and reticular zones was measured. Factor analysis (principal component method) was implemented with the program Statistica 5.5.

Results. It was found that the dispersion of the information field of 106 parameters of the neuro-endocrine-immune complex and metabolism is absorbed by 20 principal component (PCs). Using the Cattel method, the number of PCs analyzed is limited to twelve, the total contribution of which to the total variance of the raw data is $66,2 \%$, ie, it reaches the required critical level (2/3).

We have found that there is a general pattern - the combination of neuro-endocrine and immune parameters in 8 individual PCs, which, in our material, illustrates the correctness of the concept of a triple neuroendocrine-immune complex, the elements of which carry out bilateral interaction. On the other hand, in $10 \mathrm{PCs}$, neuro-endocrine and/or immune parameters are combined with metabolism parameters. The cause/effect relationships between them will be the subject of a subsequent study.

Key words: neuroendocrine-immune complex, metabolism, factor analysis, female rats. 